\title{
Delirium in older adults, if you think it's the urine, think again
}

\author{
Rebecca Schonnop ${ }^{1,3} \cdot$ Rob Woods $^{2}$ (D) $\cdot$ Brittany Ellis ${ }^{2}$ (])
}

Received: 9 November 2021 / Accepted: 16 November 2021

(C) The Author(s), under exclusive licence to Canadian Association of Emergency Physicians (CAEP)/ Association Canadienne de Médecine d'Urgence (ACMU) 2021

Emergency departments (ED) play an important role in the care of older adults. While there are many presentations for which older adults are seen in the ED, urine studies are commonly (almost reflexively) included in the work-up for these patients regardless of urinary symptoms. In this issue of CJEM, Laguë et al. [1] published a cross-sectional survey of the current practice of Canadian physicians regarding investigations and treatment of asymptomatic bacteriuria in older patients. The investigators should be applauded for their efforts to draw attention to Canadian physician practice patterns on this important clinical topic. It revealed heterogeneity in practice patterns on urine investigations in older adults and when patients are treated with antibiotics. $79.4 \%$ of participants reported to frequently request a urine dipstick or urinalysis in patients with delirium and $52.4 \%$ immediately order a urine culture with the urinalysis. Furthermore, if bacteriuria was found in a patient with delirium who was afebrile and had no urinary symptoms, $38 \%$ of physicians immediately treated with antibiotics [1]. Treatment of asymptomatic bacteriuria in older patients with delirium has failed to demonstrate benefit and comes with risk [2]. While some participants felt uncomfortable leaving bacteriuria untreated in patients with delirium, it is important to recognize the potential harm in unnecessary treatment, as well. Adverse outcomes include, but are not limited to, significant drug interactions, premature closure on identifying the precipitating factors for delirium which distract clinicians from recognizing the true cause, antimicrobial resistance, and Clostridium difficile infections [2, 3].

\section{Rebecca Schonnop}

schonnop@ualberta.ca

1 Department of Emergency Medicine, University of Alberta, Edmonton, AB, Canada

2 Department of Emergency Medicine, University of Saskatchewan, Saskatoon, SK, Canada

3 Kingsway Emergency Agency, Royal Alexandra Hospital, \#541-5th Floor CSC Building, 10240 Kingsway Avenue NW, Edmonton, AB T5H 3V9, Canada
There was considerable variation among study participants on the definition of asymptomatic bacteriuria, with $47 \%$ of respondents defining bacteriuria in an afebrile patient with new onset of confusion and no urinary or systemic signs or symptoms incorrectly as a urinary tract infection, rather than asymptomatic bacteriuria [1]. Asymptomatic bacteriuria is clearly defined in the literature as bacteriuria [isolation of urinary pathogen(s) at $\geq 10^{5} \mathrm{CFU} / \mathrm{mL}$ in midstream urine] in the absence of genitourinary signs or symptoms. This is common in older adults, with prevalence in community dwelling older males of $4-19 \%$ and $11-16 \%$ in females as reported in this publication. However, the prevalence is even greater among persons in long-term care with up to $50 \%$ of people having asymptomatic bacteriuria, and those persons with indwelling catheters having a prevalence of $100 \%$ [4]. Previous infectious disease and microbiology guidelines have been published on this topic and support that routine urinalysis should not be sent for older adults in the ED. In the ED, urinary studies should only be sent if there are focal urinary signs or symptoms or as part of the work-up for a patient with sepsis without a localizing source. The presence of delirium, falls, and behavioral changes are not sole indications for investigation of a UTI [3, 4]. Before delirium is attributed to a UTI, assessment for other common causes should be complete, for example untreated pain, constipation, and medication side effects, followed by careful observation. These are the guidelines and recommendations that we should currently be following in clinical practice in the ED.

The aging demographic we are seeing in Canada and around the world has warranted increased attention as to how to care for older people better and more effectively in the ED. This has increased interest from researchers, special interest groups, quality improvement specialists, and clinicians to explore common themes and presentations. Asymptomatic bacteriuria is common in older adults, both community dwelling, as well as in care. Delirium alone is not a symptom of a UTI [4]. And, delirium without an identified source is an acceptable diagnosis in the ED. Given the 
increased morbidity and mortality of discharging patients with delirium without an identified source [5], admission for ongoing observation and assessment is an acceptable and appropriate treatment plan; during which time inpatient providers can determine if a urinalysis may be warranted.

It is understandable how we found ourselves in this situation. Older adults with delirium require more time to do an assessment. History often requires reaching out to collateral sources which may not be immediately available, and the physical exam may not reflect the underlying disease process. There is constant pressure to get patients seen, diagnosed, treated, and admitted or discharged. There is peer pressure from our colleagues who admit these patients, as well as pressure from patients and their families to have a diagnosis; if we do not order urine investigations we can be seen as 'not doing our job well'. The path of least resistance in an older patient with delirium is to send off a urine sample, find an abnormal urinalysis (often a foregone conclusion), and label it as a UTI. Then, we pat ourselves on the back and call Internal Medicine, despite clear guidelines cautioning against this diagnostic practice [4]. This can become a vicious cycle where the patient then gets labeled as 'frequent UTIs that present with delirium' and it becomes increasingly more difficult to hold off on ordering urine investigations and administering antibiotics.

Delirium without a clear source is a clinical challenge where asymptomatic bacteriuria is commonly and inappropriately blamed, leading to significant variation in practice. Leaving it to individual clinicians to be aware of and follow guidelines established outside the ED has led to many patients being over-investigated and over-treated, leading to increased morbidity for patients and health care costs for the system. If we want to move the needle in the direction of better care for this population, we will need the collective efforts from clinicians, researchers, quality improvement specialists, special interest groups, as well as patients and families to tackle it. Only then will we have a 'culture change'.

\section{Declaration}

Conflict of interest The authors declare that they have no conflict of interest.

\section{References}

1. Laguë A, Boucher V, Joo P, Yadav K, Morasse C, Émond M. Investigation and treatment of asymptomatic bacteriuria in older patients with delirium: a cross-sectional survey of Canadian physicians. Can J Emerg Med. 2021. https://doi.org/10.1007/ s43678-021-00148-1.

2. Dasgupta M, Brymer C, Elsayed S. Treatment of asymptomatic UTI in older delirious medical in-patients: a prospective cohort study. Arch Gerontol Geriatr. 2017;72:127-34.

3. Woods R, Schonnop R, Henschke S, Ellis B. Just the facts: Diagnosis and treatment of urinary tract infections in older adults. Can J Emerg Med. 2021. https://doi.org/10.1007/s43678-021-00131-w.

4. Nicolle LE, Gupta K, Bradley SF, Colgan R, DeMuri GP, Drekonja $\mathrm{D}$, et al. Clinical practice guideline for the management of asymptomatic bacteriuria: 2019 update by the Infectious Diseases Society of America. Clin Infect Dis. 2019;68(10):e83-110.

5. McAvay GJ, Van Ness PH, Bogardus ST Jr, Zhang Y, Leslie DL, Leo-Summers LS, et al. Older adults discharged from the hospital with delirium: 1-year outcomes. J Am Geriatr Soc. 2006;54(8):1245-50. 